\author{
Methodik StrR \\ Übungsklausur
}

Richter Dr. Joachim Eiden

\title{
Vom falschen Schwimmlehrer, der lieber baden ging
}

Joachim Eiden: Der Autor ist Richter am Amtsgericht in Wolfratshausen bei München. Zur Zeit der Klausurstellung war er wissenschaftlicher Mitarbeiter bei Prof. Dr. Petra Wittig, Professur für Strafrecht und Rechtsphilosophie an der LMU München.

Stichworte: Urkundendelikte - Anstiftung - Anstellungsbetrug - Rücktritt bei mehreren Beteiligten

Die Klausur wurde in leicht abgewandelter Form im Sommersemester 2011 an der Ludwig-Maximilians-Universität München im Grundkurs Strafrecht als Zwischenprüfungsklausur gestellt. Schwerpunkte der Klausur sind v.a. Urkundendelikte in verschiedensten Varianten sowie das Problem des Anstellungsbetruges.

\section{SACHVERHALT}

Svenja (S) und Bernhard (B) sind seit mehreren Jahren verheiratet. S, die als Informatikerin in einem Großraumbüro in München tätig ist, hat keine Lust mehr, allein für den Lebensunterhalt der beiden arbeiten zu müssen, während B den ganzen Tag nur an der Isar liegt und zwischen Sonnen und Baden hin und her wechselt. Ständig predigt sie ihrem Mann, dass sie von ihm etwas mehr Engagement erwarte, immerhin habe er ein abgeschlossenes BWL-Studium und mit BWL könne man doch schließlich alles machen.

An einem Montag Morgen drückt sie ihm den Stellenanzeigenteil der Lokalzeitung in die Hand und weist ihn mit den Worten »Da kannst Du das Angenehme mit dem Nützlichen verbinden « auf folgendes Inserat hin:

»Gesucht wird ein/e Schwimmlehrer/in, die/der ihre/ seine erfolgreiche Ausbildung durch ein Zertifikat einer staatlich anerkannten Schwimmausbildungsschule nachweist. Tätigkeiten: Unterricht von Nichtschwimmern jeden Alters im Freibad F. Bezahlung: Pauschale je nach Kursdauer, mind. $200 €$. Bewerbungen bitte unter..."

$B$ ist darüber nicht erbaut, vor allem da er nach einer Recherche im Internet feststellen muss, dass der Erwerb eines Zertifikates ihn mindestens 6 volle Tage kosten und in der Schwimmhalle stattfinden würde. Außerdem hat der Wetterbericht für die nächste Woche eine stabile Hochdrucklage mit sonnigen $30^{\circ} \mathrm{C}$ angekündigt. Als er dies alles am Abend der völlig erschöpften S mitteilt, sagt diese nur: "Schatz, wir haben einen Farb-Laser-Drucker, letzten Winter hast Du ein Grafik-Seminar besucht und Unterschriften fälschen konntest Du schon in der Kollegstufe.« Dabei geht $\mathrm{S}$ davon aus, dass B zu faul ist, das Zertifikat redlich zu erwerben.

B geht nicht nur langsam seine Frau auf die Nerven, er findet überraschenderweise auch seinen sportlichen Ehrgeiz geweckt: Es könne ja nicht so schwierig sein, ein Zertifikat zu basteln, denkt er, und so ein bisschen Unterricht im Freibad, das ließe sich schon machen.

Noch am selben Abend setzt er sich an den Computer und erstellt aus dem Adresskopf einer Schwimmschule, die er im Internet findet, ein perfektes Teilnahme-Zertifikat und kopiert seinen Namen hinein; das ausgedruckte Dokument unterschreibt er mit dem Namen des Schwimmschulinhabers.

Am nächsten Tag schickt er seine Bewerbungsunterlagen samt Zertifikat an die in der Annonce angegebene Adresse, um damit seine Eignung kund zu tun und seine Chancen zu verbessern. Dabei weiß er, dass er ganz ordentlich schwimmen kann, aber keine Ahnung hat, wie er optimal Schwimmunterricht erteilen muss und es somit ausgeschlossen ist, Nichtschwimmern qualifiziert die Grundlagen des Schwimmens beizubringen.

Wider Erwarten erhält er wenige Tage später eine Einladung zum Vorstellungsgespräch, wovon er seiner Frau freudig berichtet. S indessen hat Skrupel bekommen, ob das alles so eine gute Idee war und teilt ihre Skepsis B mit. Dieser ist empört über die Wankelmütigkeit seiner Frau und beharrt auf dem Vorstellungsgespräch. S, die keine Lust auf Streit hat, lässt die Sache auf sich beruhen, in der Gewissheit, ihr Mann würde die Stelle wegen offenkundiger Unlust sowieso nicht erhalten.

Allerdings kommt es anders, denn B erhält nach dem Vorstellungsgespräch aufgrund seiner vermeintli- 
chen Qualifikation die Stelle zu folgenden Bedingungen: Gegen eine Pauschale von $500 €$ muss B einen 3-tägigen Schwimmkurs mit 15 Teilnehmern geben.

Als B seine Arbeit antreten soll, hat er plötzlich keine Lust mehr; das Geld möchte er dagegen schon gerne bekommen und hofft, dass sein Arbeitgeber es trotzdem bezahlt. Kurzerhand meldet er sich, obwohl rundum gesund, in der Früh krank und legt sich gemütlich an die Isar. $\mathrm{S}$ weiß davon nichts. Am Ende des Monats werden ihm die vollen $500 €$ überwiesen, weil der Arbeitgeber irrtümlich meint, wegen der Krankheit zur Lohnfortzahlung verpflichtet $\mathrm{zu}$ sein. B behält das Geld freudig ein und kauft sich davon eine neue Designer-Badehose.

\section{Bearbeitervermerk:}

Wie haben sich B und S nach dem StGB strafbar gemacht?

Evtl. Strafanträge sind gestellt.

\section{LÖSUNGSVORSCHLAG:}

\section{Tatkomplex: Das »Zertifikat»}

\section{STRAFBARKEIT DES B :}

\section{A. Strafbarkeit gemäß § 267 I Alt. 1 StGB durch Erstellen des "Zertifikats" am Bildschirm ${ }^{1}$}

Indem B am Bildschirm des Computers das "Zertifikat" erstellte, könnte er sich wegen Urkundenfälschung gemäß $\S 267$ I Alt. 1 StGB strafbar gemacht haben.

\section{Tatbestandsmäßigkeit}

\section{Objektiver Tatbestand}

Die Verwirklichung des objektiven Tatbestandes setzt zunächst das Vorliegen einer Urkunde voraus, was hinsichtlich des »Zertifikats « auf dem Bildschirm fraglich ist. Eine Urkunde ist die verkörperte (d. h. mit einer Sache fest verbundene), allgemein oder für Eingeweihte verständliche, menschliche Gedankenerklärung (»Perpetuierungsfunktion«), die geeignet und bestimmt ist, im Rechtsverkehr

1 Ebenso vertretbar ist es, wenn nicht das Erstellen isoliert betrachtet wird, sondern innerhalb der Prüfung einer Urkundenfälschung das Kopieren, Ausdrucken, Unterschreiben und Versenden als Einheit gesehen und einheitlich geprüft wird.
Beweis zu erbringen (»Beweisfunktion«), und ihren Aussteller (den Erklärenden) erkennen lässt (»Garantiefunktion $\ll)^{2}$.

Folglich reicht das bloße Vorhandensein des »Zertifikats« auf dem Bildschirm dafür nicht aus, denn es fehlt insoweit noch an der notwendigen Verkörperung, das virtuelle Bild ist gerade keine fest und dauerhafte Sachverbindung.

Zudem mangelt es an der Beweiseignung, kann das "Zertifikat" doch in dieser Form jedenfalls bei der Überzeugungsbildung nicht mitbestimmend ins Gewicht fallen ${ }^{3}$.

\section{Zwischenergebnis}

Da die bloße Darstellung auf dem Bildschirm noch keine Urkunde im Sinne des § 267 I StGB darstellt, ist bereits der objektive Tatbestand einer Urkundenfälschung nicht erfüllt.

\section{Ergebnis}

Mangels Vorliegen einer Urkunde hat sich B nicht gemäß $\S 267$ I Alt. 1 StGB strafbar gemacht.

\section{B. Strafbarkeit gemäß § 269 | StGB durch das Erstellen des "Zertifikats« am Bildschirm}

Durch das Erstellen des »Zertifikats« am Bildschirm könnte sich B aber wegen Fälschung beweiserheblicher Daten gemäß § 269 I StGB strafbar gemacht haben.

\section{Tatbestandsmäßigkeit}

\section{Objektiver Tatbestand}

Dazu müsste sich B Daten bedient haben, die zudem beweiserheblich sind.

\section{a) "Daten"}

Daten sind codierte, auf einen Datenträger fixierte Informationen über eine außerhalb des verwendeten Zeichen-

2 SSW-Wittig, StGB, 2009, § 267 Rn. 7 m.w. N.; Kindhäuser, BT I, 5. Aufl., 2012, §55 Rn. 8.

3 Wessels/Hettinger, BT 1, 36. Aufl., 2013, Rn. 796. 
systems befindliche Wirklichkeit ${ }^{4}$. Erfasst werden hauptsächlich Computerdaten, die in Speichermedien gesichert, in Computern verarbeitet oder in Netzwerken übertragen werden ${ }^{5}$. Da sich der Adresskopf als codierte und fixierte Information auf einem Datenträger (einem Server, der ans Internet angeschlossen ist) befindet, ist das Tatbestandsmerkmal erfüllt.

\section{b) "beweiserheblich"}

Die Daten müssten jedoch auch »beweiserheblich« sein. Beweiserheblich sind Daten, wenn sie dazu bestimmt sind, bei ihrer Verarbeitung im Rechtsverkehr als Beweisdaten für rechtlich erhebliche Tatsachen benutzt zu werden ${ }^{6}$ und sie elektronisch, magnetisch oder sonst nicht unmittelbar wahrnehmbar gespeichert werden bzw. bei Tatbegehung schon entsprechend gespeichert sind ${ }^{7}$. Aus der engen Anlehnung von $\S 269$ an $\S 267$ StGB ergibt sich, dass die gespeicherten oder veränderten Daten die Garantiefunktion des Urkundenbegriffs erfüllen müssen. Es muss also im Falle der Wahrnehmung der Urkunde deren (scheinbarer) Aussteller erkennbar sein ${ }^{8}$. Dabei ist Aussteller eines beweiserheblichen Datums ähnlich wie bei der Urkunde, wer als geistiger Urheber der Gedankenerklärung erscheint ${ }^{9}$. Die Daten müssen in einer Gesamtschau zumindest einen eigenständigen Beweiswert haben ${ }^{10}$. Das alles liegt hinsichtlich des Adresskopfes nicht vor, für sich genommen hat dieser noch keinen eigenständigen Beweiswert.

\section{Zwischenergebnis}

Da keine beweiserheblichen Daten vorlagen, die B hätte verändern können, liegt schon der objektive Tatbestand des $\S 269$ StGB nicht vor.

\section{Ergebnis}

B hat sich durch das Erstellen des »Zertifikats« am Bildschirm keiner Fälschung beweiserheblicher Daten gemäß $\S 269$ I StGB strafbar gemacht.

4 Fischer, StGB, 60. Aufl., 2013, § 268 Rn. 6 m. w. N.

5 Spindler/Schuster/Gercke, Recht der elektronischen Medien,

2. Aufl., 2011, § 269 StGB, Rn. 3.

6 SSW-Hilgendorf, StGB, 2009, § 269 Rn. 4.

7 Fischer, StGB, 60. Aufl., 2013, § 269 Rn. 4.

8 Schönke/Schröder/Cramer/Heine, 28. Aufl., 2010, § 269 Rn. 11.

9 SSW-Hilgendorf, StGB, 2009, § 269 Rn. 6.

10 Buggisch, NJW 2004, 3519, 3520.

\section{Strafbarkeit gemäß § 267 I Alt. 1 StGB durch das Ausdrucken des "Zertifikats ${ }^{11}$}

B könnte sich, indem er das »Zertifikat« ausdruckte, einer Urkundenfälschung gemäß $§ 267$ I Alt. 1 StGB strafbar gemacht haben.

\section{Tatbestandsmäßigkeit}

Zwar erfüllt der Ausdruck bereits das Erfordernis der Verkörperung, da der Gedankeninhalt jetzt als gedruckte Schrift fest mit dem Blatt Papier verbunden ist. Allerdings erfüllt der Ausdruck noch nicht die Garantiefunktion, denn mangels Unterschrift kann ihm noch keine Eignung zur Täuschung im Rechtsverkehr beigemessen werden ${ }^{12}$.

\section{Ergebnis}

Durch das bloße Ausdrucken des Zertifikats hat sich B keiner Urkundenfälschung gemäß § 267 I Alt. 1 StGB strafbar gemacht.

\section{Strafbarkeit gemäß § 267 I Alt. 1 StGB durch Unterschreiben des »Zertifikats»}

B könnte sich aber einer Urkundenfälschung nach § 267 I Alt. 1 StGB strafbar gemacht haben, als er das "Zertifikat» unterschrieb.

\section{Tatbestandsmäßigkeit}

1. Objektiver Tatbestand

a) Das ausgedruckte und unterschriebene "Zertifikat» als Urkunde

aa) "Urkunde»

Dazu müsste B eine unechte Urkunde hergestellt haben.

11 Eine Differenzierung nach Erstellen des Zertifikats am Bildschirm und Ausdrucken des Zertifikats wurde nicht als zwingend erforderlich angesehen.

12 Eine eigenhändige Unterschrift ist allerdings nicht stets erforderlich, sofern nicht unter dem Gesichtspunkt der Beweiseignung eine eigenhändige Unterschrift zur Wirksamkeit gesetzlich vorgeschrieben ist. Es genügt vielmehr, dass die Individualisierung des Ausstellers aus der Urkunde möglich ist (vgl im Einzelnen Schönke/Schröder/ Cramer/Heine, 28. Aufl., 2010, § 267 Rn. 17 m. w. N.). Diese Individualisierung ist im Falle eines Zertifikates jedoch nur mittels Unterschrift eindeutig möglich. 
Das ausgedruckte »Zertifikat« zeigt dabei die notwendige Verkörperung, da die gedruckten Buchstaben fest mit dem Blatt Papier verbunden sind (=Perpetuierungsfunktion). Des Weiteren ist nun auch die Garantiefunktion gegeben: Nach der sog. „Geistigkeitstheorie« ist Aussteller diejenige Person, der die urkundliche Erklärung im Rechtsverkehr als Urheber zuzurechnen ist ${ }^{13}$. Wer Aussteller ist bestimmt sich nach dem Empfängerhorizont ${ }^{14}$ : ein Außenstehender wird das "Zertifikat" als von dem Schwimmschulinhaber ausgestellt werten.

Zuletzt ist auch die Beweisfunktion erfüllt, denn nun ist das "Zertifikat« sowohl dazu bestimmt, als auch dazu geeignet, Beweis zu bringen über die Tatsache, dass der Schwimmschulinhaber dem B die im »Zertifikat« beschriebenen Qualifikationen bescheinigt. Das Zertifikat kann damit für denjenigen, dem es vorgelegt wird, bei der Bildung seiner Überzeugung, ob er den Vorlegenden einstellen soll oder nicht, mitbestimmend sein, und diese Möglichkeit reicht aus. Eine Urkunde liegt damit vor.

\section{bb) "Herstellen einer unechten Urkunde»}

\section{i. S. d. § 267 I Alt. 1 StGB}

B müsste damit auch eine unechte Urkunde hergestellt haben.

Herstellen einer unechten Urkunde ist das Ausstellen mit dem Ansehen, als sei sie von einer anderen Person ausgestellt, mithin echt (Identitätstäuschung) ${ }^{15}$. B hat durch die Verwendung des Adresskopfes und die Unterschrift des Schwimmschulinhabers dem Zertifikat den Anschein gegeben, als würde es tatsächlich von diesem stammen. Der Fall liegt damit so wie bei einem für die Benutzung eines durch Fotomontage hergestellten fremden Briefkopfbogens. Auch hierbei verändert der Täter keine vorhandene Urkunde, sondern er stellt eine neue Urkunde her und täuscht hierbei über die Person des Ausstellers ${ }^{16}$. B hat folglich eine unechte Urkunde hergestellt.

\section{b) „Verfälschen«i i. S. d. § 267 I Alt. 2 StGB}

Ein Verfälschen einer echten Urkunde gemäß § 267 I Alt. 2 StGB liegt dagegen nicht vor, denn es existierte zuvor gerade keine Urkunde, die B hätte verfälschen können.

\section{Subjektiver Tatbestand a) Vorsatz}

B müsste auch vorsätzlich gehandelt haben. Der Vorsatz muss neben der Tathandlung die Merkmale umfassen, die die Urkundeneigenschaft begründen, wobei bedingter Vorsatz ausreicht ${ }^{17}$. Dabei reicht bei normativen Tatbestandsmerkmalen wie »Urkunde« aus, wenn der Täter den rechtlich sozialen Bedeutungsgehalt des Tatumstandes nach Laienart erfasst hat, sog. Parallelwertung in der Laienssphäre $^{18}$. B wusste, dass er ein Schriftstück erstellte, das einen bestimmten (anderen) Aussteller erkennen lässt und das zum Beweis seiner vermeintlichen Qualifikation dienen sollte.

\section{b) "Zur Täuschung im Rechtsverkehr»}

B müsste aber auch »zur Täuschung im Rechtsverkehr» gehandelt haben und zwar bereits beim Fälschen; hierbei reicht "sicheres Wissen" (dolus directus 2. Grades) aus ${ }^{19}$. Es genügt nicht, über die Echtheit der Urkunde täuschen zu wollen; vielmehr muss der Täter mittels der Urkunde im Rechtsverkehr täuschen wollen, d. h. einen Irrtum erregen und dadurch ein rechtlich erhebliches Verhalten erreichen wollen ${ }^{20}$.

B hatte schon beim Erstellen der Urkunde Absicht zur Täuschung im Rechtsverkehr. Er stellte sie nur her, um damit im Zuge seiner Bewerbung eine Einstellung zu erreichen.

\section{Rechtswidrigkeit}

Es sind keine Rechtfertigungsgründe ersichtlich. B handelte damit auch rechtswidrig.

\section{Schuld}

Entschuldigungs- bzw. Schuldausschließungsgründe sind in der Person des B nicht ersichtlich, so dass ihm die Tat auch persönlich vorzuwerfen ist.
13 Kindhäuser, LPK-StGB, 5. Aufl., 2013, § 267 Rn. 5.

14 Fischer, StGB, 60. Aufl., 2013, § 267 Rn. 11.

15 SSW-Wittig, StGB, 2009, § 267 Rn. 62.

16 Fischer, StGB, 60. Aufl., 2013, § 267 Rn. 20 m. w. N.; vgl. auch OLG

Zweibrücken NJW 1982, 2268.
17 Lackner/Kühl StGB, 27. Aufl., 2011, § 267 Rn. 23 m. w. N.

18 Wessels/Beulke, AT, 42. Aufl., 2012, Rn. 243.

19 SSW-Wittig, StGB, 2009, § 267 Rn. 84.

20 Fischer, StGB, 60. Aufl., 2013, § 267 Rn. 42. 


\section{Ergebnis}

Durch das Unterschreiben des »Zertifikats« hat sich B gemäß § 267 I Alt. 1 StGB strafbar gemacht.

\section{E. Strafbarkeit gemäß § 267 I Alt. 3 StGB durch Versenden der Bewerbungsunterlagen samt »Zertifikat«}

Indem B die Bewerbungsunterlagen samt »Zertifikat« absendete, könnte er sich einer Urkundenfälschung gemäß $\S 267$ I Alt. 3 StGB strafbar gemacht haben.

\section{Tatbestandsmäßigkeit}

\section{Objektiver Tatbestand}

a) Wie bereits erörtert, stellt das von B gefertigte Zertifikat eine unechte Urkunde dar

b) Fraglich ist, ob B durch das Versenden diese Urkunde auch "gebraucht" hat i. S. d. \$267 I Alt. 3 StGB

Gebrauchen der Urkunde bedeutet, sie der sinnlichen Wahrnehmung zugänglich zu machen, sei es durch Vorlegen, Übergeben, Hinterlegen ${ }^{21}$. Gebrauchen setzt voraus, dass der zu Täuschende in die Lage versetzt wird, von der Urkunde Kenntnis zu nehmen ${ }^{22}$.

Durch das Versenden der Bewerbungsunterlagen und das Eingehen dieser beim Empfänger, wird diesem auch das gefälschte Zertifikat zur sinnlichen Wahrnehmung zugänglich gemacht. Ein Gebrauchen liegt demnach vor.

\section{Subjektiver Tatbestand}

a) Vorsatz

B handelte vorsätzlich, denn es ging ihm darum, die Bewerbungsunterlagen dem Empfänger zur Kenntnis zu bringen.

\section{b) "Zur Täuschung im Rechtsverkehr"}

Zusätzlich müsste B aber auch zur Täuschung im Rechtsverkehr gehandelt haben. B wollte mittels der Urkunde im Rechtsverkehr täuschen, d.h. einen Irrtum erregen und

21 BeckOK-StGB/Weidmann, 21. Ed., § 267 Rn. 27; im Einzelnen MünchKomm-StGB/Erb, 2006, § 267 Rn. 195 ff.

22 Fischer, StGB, 60. Aufl., 2013, § 267 I Rn. 36 m. w. N.; Schönke/ Schröder/Cramer/Heine, 28. Aufl., 2010, § 267 Rn. 73. dadurch ein rechtlich erhebliches Verhalten, nämlich seine Einstellung, erreichen ${ }^{23}$.

\section{Rechtswidrigkeit}

Es sind keine Rechtfertigungsgründe ersichtlich. B handelte damit auch rechtswidrig.

\section{Schuld}

B handelte auch schuldhaft.

\section{Strafzumessung}

Ein besonders schwerer Fall der Urkundenfälschung durch Verwirklichung des Regelbeispiels der »Gewerbsmäßigkeit» gemäß § 267 III 2 Nr. 1 Alt. 1 StGB kommt nicht in Betracht, da der Sachverhalt insoweit keine Angaben über eine entsprechende Zukunftsmotivation des B enthält ${ }^{24}$.

\section{Ergebnis}

B hat sich durch das Versenden der Bewerbungsunterlagen samt "Zertifikat« einer Urkundenfälschung gemäß §267 I Alt. 3 StGB strafbar gemacht.

\section{Konkurrenzen ${ }^{25}$}

Da B sowohl § 267 I Alt. 1 StGB als auch § 267 I Alt. 3 StGB verwirklicht hat, stellt sich die Frage nach dem Verhältnis dieser Begehungsformen des $\S 267$ StGB zueinander ${ }^{26}$.

Hat der Täter bei der Fälschungshandlung bereits den späteren Gebrauch nicht nur umrisshaft sondern in be-

23 Fischer, StGB, 60. Aufl., 2013, § 267 Rn. 42.

24 A. A. schwer vertretbar, allenfalls mit dem Hinweis darauf, dass es mit einem einmaligen Beitrag des B zur gemeinsamen Lebensfinanzierung nicht getan sei und $\mathrm{B}$ daher eine wiederholte Tatbegehung anstrebe.

25 Eine Auseinandersetzung mit dem Verhältnis beider Begehungsformen war zwingend erforderlich, konnte jedoch auch bei den Konkurrenzen am Schluss erörtert werden.

$26 \mathrm{Zu}$ diesem Streit mit allen seinen Facetten NK-Puppe, 3. Aufl., 2010, § 267 Rn. 108 m. w. N.; vgl. auch Freund, Urkundenstraftaten, 2. Aufl. 2010, Rn. 230 m. w. N. 
stimmter Weise geplant, so bildet der Fälschungsakt und der Gebrauch des Falschstückes nur eine Straftat ${ }^{27}$. Es liegt damit nur eine Urkundenfälschung vor ${ }^{28}$.

\section{STRAFBARKEIT DER S:}

\section{A. Strafbarkeit gemäß $\S \S 267$ I Alt. 1, 26 StGB wegen Anstiftung zur Urkundenfälschung ${ }^{29}$}

Indem S dem B die Stellenanzeige mit den Worten "Schatz, wir haben einen Farb-Laser-Drucker, letzten Winter hast Du ein Grafik-Seminar besucht und Unterschriften fälschen konntest Du schon in der Kolleg-Stufe!« übergab, könnte sie sich einer Anstiftung zur Urkundenfälschung gemäß §§ 267 I Alt. 1, 26 StGB strafbar gemacht haben.

\section{Tatbestandsmäßigkeit}

\section{Objektiver Tatbestand}

Dazu müsste eine vorsätzliche rechtswidrige Haupttat des B vorliegen, zu der S den B bestimmt hat ( $\$ 26 \mathrm{StGB})$.

\section{a) "vorsätzliche rechtswidrige Haupttat"}

Die vorsätzliche rechtswidrige Haupttat des B ist seine Urkundenfälschung gemäß § 267 I Alt. 1 StGB.

\section{b) "Bestimmen"}

Fraglich ist aber, ob die S den B hierzu bestimmt hat. Wann ein Bestimmen i. S. d. § 26 StGB vorliegt, ist umstritten.

aa) Nahezu Einigkeit besteht aber insoweit, als ein zur Ausführung einer konkreten Tat bereits Entschlossener (sog. omnimodo facturus) nicht mehr angestiftet werden $\mathrm{kann}^{30}$. Da B bis zu diesem Zeitpunkt gar nicht daran

27 LK-Zieschang, 12. Aufl., §267 Rn. 287; Rengier BT II, 12. Aufl., 2011, $\S 33$ Rn. 37.

28 SSW-Wittig, StGB, 2009, § 267 Rn. 91. (a. A. vertretbar, wenn man von zwei Urkundenfälschungen ausgeht. Dann muss man sich aber mit dem Verhältnis Vortat/Nachtat auseinandersetzen; vgl. dazu eingehend Schönke/Schröder/Cramer/Heine, 28. Aufl., 2010, § 267 Rn. 79 a ff. m. w. N.

29 Als vertretbar wurde es angesehen, wenn kurz auf § 25 II StGB eingegangen, dieser aber abgelehnt wurde, da dessen Voraussetzungen mangels Tatherrschaft nicht vorliegen.

30 Kindhäuser, AT, 3. Aufl. 2008, § 41 Rn. 11 m. w. N. gedacht hatte, ein Zertifikat zu fälschen, war er noch in keiner Weise zu einer konkreten Urkundenfälschung entschlossen. Erst durch die Kombination von Inserat und den Worten der S entstand in ihm der Tatentschluss, sich ein Zertifikat zu basteln. Wenn B auch dann seinen sportlichen Ehrgeiz geweckt sah und aus eigenem Antrieb weitermachte, so war er in dem Zeitpunkt, als S die fraglichen Worte an ihn richtete und ihm das Inserat übergab, nicht einmal auch nur tatgeneigt.

bb) Meinungsstand zum »Bestimmen $\aleph^{31}$.

Nach Ansicht der Rechtsprechung und Teilen der Literatur bedeutet Bestimmen das Hervorrufen des Tatentschlusses. Ausreichend ist dabei jede hierfür ursächliche Handlung (sog. Verursachungstheorie) ${ }^{32}$. Nach einer anderen in der Literatur vertretenen Ansicht, der sog. »Kommunikationstheorie« oder »Theorie des geistigen Kontaktes«, wird eine kommunikative Beeinflussung des Täters durch den Anstifter gefordert ${ }^{33}$. Danach muss zur objektiven Verursachung ein (schlüssiger oder konkludenter) Akt der Kommunikation zwischen Täter und Anstifter hinzutreten; das bloße Schaffen einer Sachlage, die einen anderen zur Begehung einer Tat nur anreizt, genügt hiernach nicht ${ }^{34}$. Nach einer noch weiter einschränkenden Ansicht soll zwischen den Beteiligten ein sog. Unrechtspakt erforderlich sein $^{35}$ : Der Anstifter muss demzufolge über die bloße »Anreizung zur Tat« eine Art Pakt mit dem Täter schließen, der zwar keine rechtliche aber eine faktische Bindung des Täters zur Folge hat, mit anderen Worten: Er muss ihm »ein Versprechen oder eine Verpflichtung zur Tat abnehmen $\aleph^{36}$. Damit steigert sich der Anteil an der Planung derart, »dass man wie beim Mittäter von einem gemeinsamen Tatplan, wenn auch nicht von einer gemeinsamen Tatausführung sprechen kann. « ${ }^{37}$

cc) Bestimmen des B durch S.

S sagte $\mathrm{zu}$ B, nachdem sie ihm das Stelleninserat übergeben hatte und B seine Unlust betreffend den ordnungsgemäßen Erwerb eines Zertifikats zum Ausdruck gebracht hatte: "Schatz, wir haben einen Farb-Laser-Drucker, letz-

31 Es reicht aus, wenn sich die Bearbeiter, angesichts der Vielzahl an Meinungen, mit den gängigen Auffassungen bezüglich des Bestimmens befassen. Zur Fülle der Ansichten, vgl. die Übersicht bei Hilgendorf, JURA 1996, $9 \mathrm{ff}$.

32 Nachweise bei Fischer, StGB 60. Aufl., 2013, § 26 Rn. 3. 33 Kühl, AT, 7. Aufl., 2012, § 20 Rn. 172 m. w. N.; Schönke/Schröder/ Heine, 28. Aufl., 2010, § 26 Rn. 4 f.

34 Kühl, AT, 7. Aufl., 2012, § 20 Rn. 171.

35 Puppe, GA 1984, 101, 112.

36 Puppe, GA 1984, 101, 112.

37 Puppe, GA 1984, 101, 112. 
ten Winter hast Du ein Grafik-Seminar besucht und Unterschriften fälschen konntest Du schon in der Kolleg-Stufe!« Außerdem ist S der Überzeugung, B sei zu faul, das Zertifikat redlich zu erwerben.

Damit hat S den B überhaupt erst auf die Idee gebracht. Das Verhalten der S war somit unmittelbar kausal für den bei B entstandenen Tatentschluss, so dass nach der sog. Verursachungstheorie ein Bestimmen durch S vorliegt. Zudem ist die Interaktion zwischen S und B als ein konkludenter Kommunikationsakt zu werten, denn aus der Gesamtschau ergab sich für B unzweifelhaft, was S vermitteln wollte, wenn sie auch nicht ausdrücklich gesagt hatte, er solle das Zertifikat fälschen. Damit liegt auch nach der sog. Kommunikationstheorie ein Bestimmen vor. Aber auch nach der engen Auffassung, die einen Unrechtspakt fordert und dabei ganz auf das die Hervorrufung des Tatentschlusses als Erfolg der Anstiftung verzichtet ${ }^{38}$, liegt ein Bestimmen vor, denn vor dem Hintergrund der familiären Situation - S finanziert betont widerwillig alleine die Partnerschaft, während B faulenzt - erscheint das Verhalten der S nicht nur als bloßer Anreiz für B, sondern durchaus als Vereinbarung. Und B, der die Aufgabe annimmt, scheint seinerseits ein konkludentes Versprechen zur Durchführung zu geben, das zeigen soll, er wolle nun auch etwas zur Finanzierung der Lebensgemeinschaft beitragen. Als B dann zur Urkundenfälschung übergeht, motiviert ihn diese Vereinbarung mit der S jedenfalls noch mit $^{39}$, neben dem eigenen Ehrgeiz, der plötzlich geweckt wurde. Auch nach der einschränkenden Lehre vom Unrechtspakt ist ein »Bestimmen« i.S.d. § 26 StGB daher gegeben $^{40}$.

38 Roxin, AT II, 2003, § 26 Rn. 73.

39 Eine Mitmotivation soll ausreichen, vgl. Puppe, GA 1984, 101, $118 \mathrm{f}$.

40 Allerdings könnte man das auch ganz anders sehen und sich auf den Standpunkt stellen, von einer Vereinbarung sei nicht auch nur im Ansatz die Rede gewesen, geschweige denn von einem Versprechen; es wurde nichts vereinbart, es wurde auch nichts versprochen. In diesem Fall wäre ein Streitentscheid erforderlich: Gegen die Unrechtspaktstheorie spricht, wie vorliegende Konstellation zeigt, dass es nahezu unmöglich ist, schon objektiv tragfähige Kriterien für einen Unrechtspakt festzulegen. Viel schwieriger ist es noch, am konkreten Fall zu werten, ob es sich dabei um einen Unrechtspakt handelt oder nicht. Ganz generell spricht aber gegen diese Theorie, dass sie den Begriff des Bestimmens und damit die Strafbarkeit der Anstiftung zu sehr einengt (vgl. Kühl AT, 7. Aufl., 2012, § 20 Rn. 173), denn das Gesetz verlangt nicht, dass der Anstifter den Täter zur Tat verpflichtet, sondern nur, dass er ihn dazu „bestimmt“. (Zur Kritik vgl. Roxin AT II, 2003, § 26 Rn. 89 m.w. N.; vgl. auch die Nachweise bei Kühl AT, 7. Aufl., 2012, § 20 Fn. 283). Hinweis: Eine derart intensive Auseinandersetzung mit der Unrechtspakt-Theorie war in der Klausur nicht verlangt.
Da somit alle Ansichten zum selben Ergebnis kommen, ist ein Streitentscheid nicht erforderlich ${ }^{41}$.

\section{Subjektiver Tatbestand}

S müsste auch vorsätzlich gehandelt haben. Die subjektiven Voraussetzungen der Anstiftung umschreibt der Begriff »doppelter Anstiftervorsatz«, denn der Vorsatz des Anstifters muss sich einmal auf die eigene Anstiftungshandlung beziehen und des Weiteren auch die Ausführung der Haupttat in ihren Grundzügen und wesentlichen Merkmalen umfassen ${ }^{42}$.

S hatte Vorsatz hinsichtlich der Urkundenfälschung des B mit allen ihren Merkmalen. Sie handelte zudem vorsätzlich hinsichtlich ihrer Anstiftungshandlung, denn sie wusste, dass ihre Worte im Zusammenspiel mit den Umständen des Einzelfalles den B zur Fälschung des Zertifikats veranlassen sollten. Außerdem war S der Überzeugung, B sei zu faul, das Zertifikat redlich zu erwerben.

\section{Rechtswidrigkeit}

In Ermangelung von Rechtfertigungsgründen handelte S auch rechtswidrig.

\section{Schuld}

S handelte auch schuldhaft.

\section{Ergebnis}

$S$ hat sich der Anstiftung zur Urkundenfälschung gemäß §§ 267 I Alt. 1, 26 StGB strafbar gemacht.

\section{B. Strafbarkeit gemäß §§ 267 | Alt. 3, 26 StGB wegen Anstiftung zur Urkundenfälschung}

S hat sich gemäß §§ 267 Alt. 3, 26 StGB wegen Anstiftung zur Urkundenfälschung strafbar gemacht, denn sie wollte

41 Anders freilich, wenn man (mit durchaus guten Gründen vertretbar) einen Unrechtspakt hier ablehnt (vgl. die vorhergehende Fn.). 42 Kindhäuser AT, 4. Aufl., 2009, § 41 Rn. 21 f.; SSW-Murmann, StGB, 2009, § 26 Rn. 8. 
von Anfang an und auch zu diesem Zeitpunkt noch, dass B sich mit dem gefälschten Zertifikat bewirbt.

Aber auch hier verdrängt $\S \S 267$ I Alt. 3, 26 StGB auf Konkurrenzebene aus denselben Gründen §§ 267 I Alt. 1, 26 StGB, wie zuvor bei B erörtert. Damit liegt auch bei S nur eine Anstiftung zur Urkundenfälschung vor.

\section{Tatkomplex: Das Vorstellungsgespräch}

\section{STRAFBARKEIT DES B:}

\section{A. Strafbarkeit gemäß § 263 I StGB zu Lasten des Arbeitgebers}

Dadurch dass B sich im Vorstellungsgespräch als zertifizierter Schwimmlehrer darstellte und so die Stelle erhielt, könnte er sich wegen Betruges gemäß § 263 I StGB strafbar gemacht haben.

\section{Tatbestandsmäßigkeit}

\section{Objektiver Tatbestand}

\section{a) Täuschung über Tatsachen}

Zunächst müsste B über Tatsachen getäuscht haben. Tatsachen sind dem Beweise zugängliche Ereignisse i.S.v. Zuständen der Gegenwart oder Vergangenheit ${ }^{43}$. Eine Täuschung über eine Tatsache kann sowohl durch ausdrückliches oder konkludentes Tun, als auch durch Unterlassen erfolgen ${ }^{44}$. Als Tatsache kommt hier die Eignung als Schwimmlehrer in Betracht. Hierbei handelt es sich um eine Qualifikation, die dem Beweise zugänglich ist. Hierüber hat $B$ getäuscht.

\section{b) Irrtum}

Spiegelbildlich zur Täuschung muss beim Getäuschten ein Irrtum erregt werden. Irrtum ist hierbei jeder Widerspruch zwischen einer subjektiven Vorstellung und der Wirklichkeit ${ }^{45}$. Bei dem Arbeitgeber entstand die Vorstellung, B habe tatsächlich die dort ausgewiesene Eignung als Schwimmlehrer.

43 Lackner/Kühl, StGB, 27. Aufl., 2011, § 263 Rn. 4; Kindhäuser, LPKStGB, 5. Aufl., 2013, § 263 Rn. 53

44 Rengier BT I, 13. Aufl., 2011, §13 Rn. 8.

45 Schönke/Schröder/Cramer/Perron, StGB, 28. Aufl. 2010, § 263 Rn. 33 m. w. N.

\section{c) Vermögensverfügung}

Schließlich verlangt $\S 263$ StGB als notwendiges Bindeglied zwischen Täuschung und Irrtum noch als ungeschriebenes Tatbestandsmerkmal eine Vermögensverfügung ${ }^{46}$. Darunter ist jedes Tun, Dulden oder Unterlassen, $\mathrm{zu}$ verstehen, das unmittelbar $\mathrm{zu}$ einer Vermögensminderung führt ${ }^{47}$. Hier ging der Arbeitgeber mit B einen Dienstvertrag ein; dies führte zu seiner Lohnzahlungsverbindlichkeit ${ }^{48}$ und damit unmittelbar zu einer Vermögensminderung.

\section{d) Vermögensschaden}

Fraglich ist jedoch, ob auch ein Vermögensschaden gegeben ist. Ein Vermögensschaden liegt vor, wenn das Vermögen in seinem Gesamtwert gemindert ist. $\mathrm{Zu}$ vergleichen ist somit der Wert des Gesamtvermögens vor der Vermögensverfügung mit dem danach vorhandenen Wert ${ }^{49}$.

Problematisch ist hier die Frage, wann genau ein Vermögensschaden entsteht, wenn sich jemand durch falsche Unterlagen eine Anstellung erschleicht, denn da es sich um einen Vertrag und folglich um ein Austauschverhältnis handelt, stellt sich die Frage einer Schadenskompensation, ob also die Vermögensminderung durch eine »unmittelbar« aufgrund der Vermögensverfügung eingetretene Gegenleistung kompensiert wurde, was in die Gesamtsaldierung einzubeziehen wäre ${ }^{50}$. Diese Konstellation wird unter dem Schlagwort »Anstellungsbetrug « diskutiert, einem Unterfall des Eingehungsbetrugs ${ }^{51}$, bei welchem ein sog. Eingehungsschaden (oder Anstellungsschaden) in Form einer schadensbegründenden Vermögensgefährdung entsteht ${ }^{52}$. Grundsätzlich tritt in dieser Konstellation ein (Gefährdungs-)Schaden ${ }^{53}$ bereits mit Abschluss des Arbeitsvertrages ein, wenn der Wert des Anspruchs auf die Arbeitsleistung des Täuschenden hinter der dafür verein-

46 SSW-Satzger, StGB, 2009, § 263 Rn. 87.

47 Kindhäuser, BT II, 7. Aufl., 2013, § 27 Rn. 40.; Fischer, 60. Aufl., 2013, § 263 Rn. 70.

48 Das Problem des Anstellungsbetruges - hier erörtert unter d) kann auch unter c) geprüft werden.

49 Haft/Hilgendorf, BT I, 9. Aufl., 2009, S. 89; Wessels/Hillenkamp BT 2, 35. Aufl., 2012, Rn. 538 m. w. N.

50 Wittig, Wirtschaftsstrafrecht, 2. Aufl., 2011, §14 Rn. 102 f.

51 NK-Kindhäuser, 3. Aufl., 2010, § 263 Rn. 322.

52 SSW-Satzger StGB, 2009, § 263 Rn. 182.

53 Hinweis: Nach neuerem Verständnis handelt es sich um einen Schaden (mit der Gefahr der Schadensvertiefung) und nicht nur um eine schadensgleiche Vermögensgefährdung, was nur eine Schadensgefahr bedeuten würde. Der Ansicht des 1. Strafsenates zufolge, wird der Begriff der schadensgleichen Vermögensgefährdung gar als entbehrlich angesehen (vgl. eingehend Wittig, Wirtschaftsstrafrecht, 2. Aufl., 2011, S. 112 ff. m. w. N.). 
barten Vergütung zurückbleibt ${ }^{54}$. Bei Erschleichung eines privatrechtlichen ${ }^{55}$ Anstellungsverhältnisses ist von dem Grundsatz auszugehen, dass eine Täuschung über Vorbildung und berufliche Erfahrung in der Regel nicht zur Begründung eines Schadens ausreicht ${ }^{56}$. Jedoch soll ein Vermögensschaden ausnahmsweise zum einen dann vorliegen, wenn der Täter außerstande ist, die erwartete Arbeitsleistung $\mathrm{zu}$ erbringen, weil er die erforderlichen Voraussetzungen von vornherein nicht erfüllt ${ }^{57}$; zum anderen trotz einer der Vergütung entsprechenden fachlichen Eignung und Arbeitsleistung ausnahmsweise auch dann, wenn die Entlohnung Anteile für besondere Qualifikationen, Vertrauenswürdigkeit oder Zuverlässigkeit des Stelleninhabers enthält, dieser aber die dafür erforderlichen Vorbedingungen nicht erfüllt ${ }^{58}$. B besitzt hier nach dem Sachverhalt offensichtlich schon nicht die Fähigkeit, Nichtschwimmern die Grundlagen des Schwimmens beizubringen. Nach dem Sachverhalt ist ein Unterrichtserfolg sogar ausgeschlossen. Damit ist ein Vermögensschaden bereits durch das Erschleichen der Anstellung gegeben.

\section{e) Kausalzusammenhang}

Schließlich besteht auch der zwischen sämtlichen Merkmalen des objektiven Tatbestandes erforderliche kausale Zusammenhang ${ }^{59}$.

\section{Subjektiver Tatbestand}

\section{a) Vorsatz}

B handelte vorsätzlich hinsichtlich aller objektiven Tatbestandsmerkmale. Hinsichtlich des Gefährdungsschadens reicht die Kenntnis der Umstände, die die Gefahr des Vermögensverlustes begründen, aus ${ }^{60}$.

54 SSW-Satzger StGB, 2009, § 263 Rn. 190.

55 Bei der Erschleichung einer Beamtenstellung ist unabhängig von der Qualität der zugesagten und geleisteten Dienste und unabhängig von falschen Angaben zu gehaltsrelevanten Faktoren ein Vermögensschaden dann zu bejahen, wenn die Ernennungsvoraussetzungen nicht vorlagen und über diese getäuscht wurde (Rengier, BT I, 13. Aufl., 2011, § 13 Rn. 225; SSW-Satzger, StGB, 2009, § 263 Rn. 194). Ausführlich zu den verschiedenen Fallgruppen NK-Kindhäuser, 3. Aufl., 2010, $\S 263 \mathrm{Rn} .323 \mathrm{ff} . \mathrm{m}$. w. N., der noch weitergehend differenziert.

56 Fischer, StGB, 60. Aufl., 2013, § 236 Rn. 152; Zu den Voraussetzungen eines Anstellungsbetrugs bei Erschleichung eines privatrechtlichen Anstellungsverhältnisses vgl. eingehend BGH NJW 1978, 2042, 2043.

57 Fischer, StGB, 60. Aufl., 2013, § 263 Rn. 152.

58 Rengier, BT I, 13. Aufl., 2011, § 13 Rn. 226; Schönke/Schröder/ Cramer/Perron, StGB, 28. Aufl., 2010, § 263 Rn. 154 m. w. N.

59 Vgl. hierzu Kindhäuser, BT II, 7. Aufl., 2013, § 27 Rn. 72 f.

60 Fischer, 60. Aufl., 2013, § 263 Rn. 182.

\section{b) Absicht stoffgleicher Bereicherung}

B müsste auch die weitergehende Absicht stoffgleicher Bereicherung gehabt haben.

aa) Die Bereicherung war für B Hauptziel geworden, insbesondere ging es ihm nicht darum, ausschließlich die S ruhig zu stellen. $\mathrm{Zu}$ beachten ist, dass sich bei der Verursachung eines Gefährdungsschadens die Absicht nicht (»spiegelbildlich«) auf Erreichung der Möglichkeit eines Vorteils richtet, sondern auf dessen Eintritt ${ }^{61}$.

bb) Aus dem Wesen des Betruges als Vermögensverschiebungsdelikt ergibt sich schließlich das ungeschriebene Tatbestandsmerkmal der "Stoffgleichheit ${ }^{62}$, wonach die erstrebte Bereicherung aus dem zugefügten Schaden stammen muss ${ }^{63}$. Diese Stoffgleichheit muss objektiv bestehen ${ }^{64}$. Dabei bezieht sich beim Forderungsbetrug die Stoffgleichheit auf den Gegenstand der Forderung $^{65}$. Da beim Anstellungsbetrug "nur« ein Gefährdungsschaden vorliegt, hat dies Konsequenzen auch für die Beschaffenheit der Stoffgleichheit ${ }^{66}$ : Stoffgleichheit ist dann gegeben, wenn dem Gefährdungsschaden des Opfers auf Seiten des Täters ein angestrebter Vorteil in Form einer identischen Chance auf Vermögensmehrung gegenübersteht ${ }^{67}$. Genau das war hier der Fall, denn durch den Abschluss des Vertrages hat B unweigerlich die Chance auf Auszahlung seines Arbeitsentgeltes erwirkt.

\section{Objektive Rechtswidrigkeit der erstrebten Bereicherung und entsprechender Vorsatz ${ }^{68}$}

Die erstrebte Bereicherung ist rechtswidrig, wenn der Täter auf sie (nach bürgerlichem oder öffentlichem Recht) keinen fälligen und einredefreien Anspruch hat ${ }^{69}$. Es ist nicht

61 Fischer, 60. Aufl., 2013, § 263 Rn. 186.; krit. MünchKomm-StGB/ Hefendehl, 2006, § 263 Rn. 718.

62 Krit. zu diesem Begriff Wessels/Hillenkamp BT 2, 35. Aufl., 2013, Rn. 588, der statt dessen von „Unmittelbarkeitsbeziehung“ spricht.

63 Rengier, BT I, 13. Aufl., 2011, §13 Rn. 246.

64 SSW-Satzger StGB, 2009, § 263 Rn. 228.

65 Kindhäuser, BT II, 7. Aufl., 2013, § 27 Rn. 79.

66 Hinweis: Sofern man nach neuerem Verständnis nicht mehr von einer schadensgleichen Vermögensgefährdung ausgeht, sondern von einem Schaden (vgl. Fn. 67), würden sich bei der Prüfung der Stoffgleichheit diese Konsequenzen nicht ergeben.

67 SSW-Satzger StGB, 2009, § 263 Rn. 233.

68 Dieses Merkmal kann auch im subjektiven Tatbestand geprüft werden, wenngleich es auch ein objektives Tatbestandsmerkmal ist. (Zum Prüfungsstandort in der Fallbearbeitung vgl. Wittig, Wirtschaftsstrafrecht, 2. Aufl., 2011, §14 Rn. 144).

69 Lackner/Kühl, StGB, 27. Aufl., 2011, § 263 Rn. 61 m. w. N. 
ersichtlich, dass B einen Anspruch auf den Vertrag, mithin auf Einstellung hat.

\section{Rechtswidrigkeit}

In Ermangelung von Rechtfertigungsgründen handelte S auch rechtswidrig.

\section{Schuld}

Es sind keine Entschuldigungs- bzw. Schuldausschließungsgründe ersichtlich, so dass B die Tat auch persönlich vorzuwerfen ist.

\section{Strafzumessung}

Auch an dieser Stelle käme das Regelbeispiel § 263 III Nr. 1 Alt. 1 StGB in Betracht. Gewerbsmäßig handelt, wer sich aus der wiederholten Tatbegehung eine fortlaufende Einnahmequelle von einigem Umfang und einer gewissen Dauer verschaffen will ${ }^{70}$.

Bei den $500 €$ handelt es sich aber um einen Pauschalbetrag und nicht um wiederkehrendes Arbeitsentgelt. Dass B die Absicht hatte, dies ein weiteres Mal zu tun, ist dem Sachverhalt nicht zu entnehmen.

\section{Ergebnis}

B hat sich wegen vollendeten Betruges gemäß § 263 I StGB strafbar gemacht.

\section{STRAFBARKEIT DER S}

\section{Gemäß §§ 263 I, 26 StGB zulasten des Arbeitgebers}

Dadurch dass S dem B die Stellenanzeige überreichte mit der Aufforderung sich dort in der soeben dargelegten Weise zu bewerben, könnte sie sich wegen Anstiftung zum Betrug gemäß §§ 263 I, 26 StGB strafbar gemacht haben.

\section{Tatbestandsmäßigkeit}

\section{Objektiver Tatbestand}

a) Eine vorsätzliche rechtswidrige Haupttat des B liegt vor, da er einen vollendeten Betrug gemäß § 263 StGB begangen hat.

b) Hierzu hat S den B in gleicher Weise bestimmt, wie zu der Urkundenfälschung, denn die Urkundenfälschung war erkanntermaßen nur notwendiger Zwischenschritt für die eigentliche Bewerbung, zu der S mit den Worten »Da kannst Du das Angenehme mit dem Nützlichen verbinden« bei B den Tatenschluss hervorrief, während sie ihm die Annonce überreichte.

\section{Subjektiver Tatbestand}

S handelte mit doppeltem Anstiftervorsatz. Dabei kann der Vorsatz des Anstifters hinsichtlich der Haupttat auch ein bedingter sein. In jedem Fall aber muss er alle Umstände umfassen, die die Strafbarkeit der Haupttat begründen ${ }^{71}$. Es genügt dabei, wenn er sich der wesentlichen Punkte der dem Angestifteten angesonnen Tat bewusst ist ${ }^{72}$. S hatte Vorsatz hinsichtlich des Betruges durch B, denn sie wusste, dass B zu faul sein würde, ein Zertifikat auf legale Weise zu erwerben. Statt dessen ging sie davon aus, B würde sich mit einem gefälschten Zertifikat bewerben, obwohl er als Schwimmlehrer völlig unqualifiziert sein würde. Außerdem wusste sie aus dem Inserat, dass als Gegenleistung ein Pauschalbetrag von mindestens $200 €$ angeboten würde. Sie handelte zudem vorsätzlich in Bezug auf ihre eigene Bestimmungshandlung.

\section{Rechtswidrigkeit}

Da keine Rechtfertigungsgründe ersichtlich sind, handelte sie auch rechtswidrig.

\section{Schuld}

Mangels Entschuldigungs- bzw. Schuldausschließungsgründen handelte $S$ auch schuldhaft. 


\section{Ergebnis}

S hat sich der Anstiftung zum Betrug gemäß §§ 263 I, 26 StGB strafbar gemacht ${ }^{73}$.

\section{Tatkomplex: Die Krankmeldung}

\section{STRAFBARKEIT DES B}

\section{Gemäß § 263 I StGB zulasten des Arbeitgebers}

Indem sich B krank meldete, ohne krank zu sein und dennoch seine Vergütung in Höhe von $500 €$ erhielt, könnte er sich wegen eines weiteren Betruges gemäß § 263 I StGB strafbar gemacht haben.

\section{Tatbestandsmäßigkeit}

\section{Objektiver Tatbestand}

\section{a) Täuschung über Tatsachen}

Zunächst müsste B über Tatsachen getäuscht haben. Tatsachen sind dem Beweise zugängliche Ereignisse i.S.v. Zuständen der Gegenwart oder Vergangenheit ${ }^{74}$.

Die Krankheit des B ist ein Zustand, der dem Beweise zugänglich ist. Hierüber täuscht $\mathrm{B}$, denn er ist in Wahrheit gesund.

\section{b) Irrtum}

Spiegelbildlich zu der Täuschung über Tatsachen müsste $B$ bei seinem Arbeitgeber einen Irrtum erregt haben. Irrtum ist jeder Widerspruch zwischen einer subjektiven Vorstellung und der Wirklichkeit ${ }^{75}$. Der Arbeitgeber glaubt, dass B tatsächlich krank ist.

73 Hinweis: Der persönliche Strafaufhebungsgrund des § 24 II 2 StGB greift hier nicht, weil es zur Vollendung der Tat kommt. Zwar hat $S$ Skrupel bekommen und teilt dem B ihre Skepsis mit. Da sie aber keine Lust auf Streit hat, lässt sie es damit bewenden. Der Anstifter ist für die vollendete Tat verantwortlich, wenn es ihm nicht gelingt, den Angestifteten von ihr abzubringen. Der Anstifter trägt wie ein Alleintäter das Risiko, dass sich der einmal hervorgerufene Tatentschluss in einer vollendeten Tat realisiert (NK-Zaczyk, 3. Aufl., 2010, § 24 Rn. 120).

74 Rengier, BT I, 13. Aufl., 2011, § 13 Rn. 4.

75 Schönke/Schröder/Cramer/Perron, StGB, 28. Aufl., 2010, § 263 Rn. 33 m.w. N.

\section{c) Vermögensverfügung}

Schließlich verlangt \& 263 I StGB noch als ungeschriebenes Tatbestandsmerkmal eine Vermögensverfügung als notwendiges Bindeglied zwischen Täuschung und Irrtum ${ }^{76}$. Unter einer Vermögensverfügung ist jedes Tun, Dulden oder Unterlassen, zu verstehen, das unmittelbar zu einer Vermögensminderung führt ${ }^{77}$.

Hier zahlte der Arbeitgeber die $500 €$ an B aus, das führt unmittelbar zu einer entsprechenden Minderung von dessen Vermögen.

\section{d) Vermögensschaden}

Des Weiteren müsste auch ein Vermögensschaden gegeben sein. Ein Vermögensschaden liegt vor, wenn das Vermögen in seinem Gesamtwert gemindert ist. $\mathrm{Zu}$ vergleichen ist somit der Wert des Gesamtvermögens vor der Vermögensverfügung mit dem danach vorhandenen Wert $^{78}$. Nach der Auszahlung ist das Gesamtvermögen des Arbeitgebers um $500 €$ gemindert, denn dieser Vermögensminderung steht kein Äquivalent als Kompensation gegenüber ${ }^{79}$. Nicht als Ausgleich zu berücksichtigen sind zudem Rückzahlungsansprüche seitens des Arbeitgebers, etwa aus ungerechtfertigter Bereicherung ${ }^{80}$, denn sie erwachsen dem Betroffenen nur mittelbar ${ }^{81}$ und setzen gerade den Eintritt eines Vermögensnachteils voraus. Im Ergebnis ist der Arbeitgeber um $500 €$ ärmer geworden. Diese $500 €$ sind ein anderer Schaden, als der im Rahmen des Anstellungsbetruges erörterte, denn er beruht auf einem neuen Täuschungsentschluss des B, den dieser erst nach Vertragsabschluss gefasst und umgesetzt hat ${ }^{82}$.

\section{e) Kausalzusammenhang}

Auch der zwischen sämtlichen Merkmalen des objektiven Tatbestandes erforderliche kausale Zusammenhang besteht $^{83}$.

76 SSW-Satzger, StGB, 2009, § 263 Rn. 87.

77 Kindhäuser, BT II, 7. Aufl., 2013, § 27 Rn. 40.; Fischer, 60. Aufl., $\S 263 \mathrm{Rn} .70$.

78 Haft/Hilgendorf, BT I, 9. Aufl., 2009, S. 93; Wessels/Hillenkamp BT 2, 34. Aufl., 2011, Rn. 538 m. w. N.

79 Es liegt auch kein Freiwerden von einer Verbindlichkeit vor, da nichts geschuldet war

80 Schönke/Schröder/Cramer/Heine, 28. Aufl., 2010, § 263, Rn. 120. 81 Wittig, Wirtschaftsstrafrecht, 2. Aufl., 2011, §14 Rn. 103. 82 Vgl. dazu Schönke/Schröder/Cramer/Perron, StGB, 28. Aufl., 2010, § 263 Rn. 137 a.E. (m.w. N. auch zur Gegenansicht).

83 Vgl. hierzu Kindhäuser, BT II, 7. Aufl., 2013, § 27 Rn. 72 f. Entgegen der h.M. fordert ein Teil der Literatur auch einen sog. funktionalen Zusammenhang zwischen Irrtum und Vermögensverfügung der- 


\section{Subjektiver Tatbestand}

\section{a) B handelte vorsätzlich}

b) Fraglich ist jedoch, ob $B$ auch die weitergehende $A b$ sicht rechtswidriger stoffgleicher Bereicherung hatte

aa) Zunächst verlangt die Bereicherungsabsicht das Streben nach einem Vermögensvorteil, dabei muss es dem Täter auf die (eigen- oder fremdnützige) Bereicherung neben anderen Zwecken - zumindest auch ankommen ${ }^{84}$. Der Bereicherungszweck muss folglich nicht alleiniger und ausschließlicher Beweggrund sein, es reicht vielmehr aus, wenn er neben einem anderen Handlungsziel steht ${ }^{85}$. Als B sich krank meldete, war sein eigentliches Ziel nur, nicht mehr zur Arbeit gehen zu müssen, allerdings hatte er die $500 €$ nach wie vor im Hinterkopf und hoffte laut Sachverhalt auf deren Zahlung, was ausreichend ist. Folglich handelte B mit Bereicherungsabsicht.

bb) Stoffgleichheit bedeutet, dass die erstrebte Bereicherung aus dem zugefügten Schaden stammen muss, sich also spiegelbildlich im geschädigten Vermögen niederschlagen muss ${ }^{86}$; dabei muss die Stoffgleichheit objektiv bestehen ${ }^{87}$. Auch das ist hier der Fall, denn hier korrespondieren Vorteil und Schaden, die angestrebten $500 €$ sind exakt die Kehrseite des Schadens ${ }^{88}$.

gestalt, dass sich der Irrtum gerade auf den schädigenden Charakter der Vermögensverfügung beziehen muss, dies mit dem Argument, dass der um das Selbstschädigende seines Handels Wissende kein Werkzeug des Täters i.S. d. Betruges sei (vgl. NK-Kindhäuser, 3. Aufl. 2010, § 263 Rn. 193 m. w. N.). Nach Kindhäuser (a. a. O., Rn. 194) soll der Täter für die Vermögensverfügung zuständig sein, wenn sich der Verfügende in einem vom Täter zu vertretenden Irrtum über Tatsachen befindet, die gerade den vermögensschädigenden Charakter seines Verhaltens betreffen, da die Verfügung andernfalls dem Täter nicht als Fremdschädigung zugerechnet werden könne. Eine Zurechnung könnte man hier jedoch bestreiten: Zwar sah sich der Arbeitgeber bei der Vermögensverfügung infolge Irrtums als unfrei an, allerdings beruhte seine Vermögensverfügung nur mittelbar auf dem Irrtum über die Krankheit des B, unmittelbar folgte sie aus seinem Irrtum über die Rechtslage in Sachen Lohnfortzahlung im Krankheitsfall. Demnach müsste man die Zuständigkeit des B für diesen Irrtum ablehnen und ihn allein der Sphäre des Arbeitgebers zuweisen, so dass eine Strafbarkeit nach $\S 263$ StGB hier mangels funktionalen Zusammenhangs ausscheiden würde.

84 Rengier, BT I, 13. Aufl., 2011, § 13 Rn. 237 f.; So soll etwa dann keine Absicht vorliegen, wenn der Täter die Bereicherung als peinliche oder lästige Folge seines Handelns hinnimmt, weil er glaubt, sonst sein anderes Zeil zu verfehlen (MünchKomm-StGB/Hefendehl, 2006, § 263 Rn. 722 m. w. N.).

85 MünchKomm-StGB/Hefendehl, 2006, § 263 Rn. 721.

86 Rengier, BT I, 13. Aufl., 2011, §13 Rn. 246.

87 SSW-Satzger StGB, 2009, § 263 Rn. 228.

88 LK-Tiedemann, 11. Aufl., 2000, § 263 Rn. 256.

\section{Objektive Rechtswidrigkeit der erstrebten Bereiche- rung und entsprechender Vorsatz ${ }^{89}$}

Die erstrebte Bereicherung ist rechtswidrig, wenn der Täter auf sie (nach bürgerlichem oder öffentlichem Recht) keinen fälligen und einredefreien Anspruch hat ${ }^{90}$. B hatte weder einen Anspruch auf Zahlung aus dem Dienstvertrag, da er seine Leistung nicht erbracht hat und daher die Einrede des nicht erfüllten Vertrages bestand. Auch eine Lohnfortzahlung im Krankheitsfall kam nicht in Betracht, zum einen war B gar nicht krank, zum anderen wäre $\S 3$ EFZG gar nicht einschlägig gewesen, da es schon mangels Arbeitnehmerstellung des B gemäß § 1 EFZG gar nicht zur Anwendung gelangt wäre. B wusste auch, dass er keinen fälligen und einredefreien Anspruch auf die $500 €$ hatte.

\section{Rechtswidrigkeit}

Da keine Rechtfertigungsgründe ersichtlich sind, handelte er auch rechtswidrig.

\section{Schuld}

Mangels Entschuldigungs- bzw. Schuldausschließungsgründen handelte er auch schuldhaft.

\section{Strafzumessung}

Der Sachverhalt enthält auch hier keine Hinweise darauf, dass B gewerbsmäßig i.S.d. § 263 III Nr. 1 Alt. 1 StGB gehandelt hat.

\section{Ergebnis}

B hat sich gemäß § 263 I StGB strafbar gemacht, indem er sich krank meldete und dennoch seine Vergütung in Höhe von $500 €$ erhielt.

89 Dieses Merkmal kann auch im subjektiven Tatbestand geprüft werden.

90 Lackner/Kühl, StGB, 27. Aufl., 2011, § 263 Rn. 61 m. w. N. 


\section{STRAFBARKEIT DER S}

Im 3. Tatkomplex hat sich S nicht strafbar gemacht, denn sie weiß hier von nichts ${ }^{91}$.

\section{GESAMTERGEBNIS:}

B hat sich wegen Urkundenfälschung gemäß § 267 I Alt. 3 $\mathrm{StGB}^{92}$ und wegen zweifachen Betruges gemäß 263 I StGB

91 Hinweis zu weiteren Tatbeständen, die von einigen Bearbeitern erörtert wurden: Ein Untreuestrafbarkeit gemäß § 266 I Alt.2 StGB scheitert bereits daran, dass der objektive Tatbestand nicht erfüllt ist, denn eine Vermögensbetreuungspflicht des B besteht nicht. § 257 StGB scheitert zum einen an der fehlenden Hilfeleistungshandlung der S zum anderen daran, dass S als Beteiligte an der Vortat strafbar ist (§ 257 III 1 StGB).

$92 \mathrm{Zu}$ den Konkurrenzen der Handlungsalternativen des § $267 \mathrm{I}$ zueinander vgl. oben 1. Tatkomplex Strafbarkeit des B. Diese Problematik hätte auch im Gesamtergebnis erörtert werden können. strafbar gemacht ${ }^{93}$. Die drei Delikte stehen in Tatmehrheit zueinander $(\S 53 \mathrm{StGB})^{94}$.

$\mathrm{S}$ hat sich wegen Anstiftung zur Urkundenfälschung gemäß §§ 267 I Alt. 3, 26 StGB und wegen Anstiftung zum Betrug gemäß §§ 263 I, 26 StGB strafbar gemacht. Beide Delikte stehen in Tatmehrheit $(\S 53 \mathrm{StGB})^{95}$.

93 Die Frage, ob die Zahlung der $500 €$ ein echter oder ein unechter Erfüllungsbetrug sind, stellt sich hier nicht, denn die Krankmeldung des B, ohne krank zu sein, war eine neue eigenständige Handlung, die nichts mehr mit der Ausgangstäuschung zu tun hatte. Anders wäre es zu beurteilen, wenn B schlecht gearbeitet und dennoch die $500 €$ kassiert hätte. (Zu typischen Konstellationen des echten- und unechten Erfüllungsbetruges und den Konsequenzen für die Konkurrenzen vgl. SSW-Satzger, StGB, 2009, § 263 Rn. 185 ff. sowie Rn. 269).

94 Es ist auch vertretbar, die Urkundenfälschung und den ersten Betrug als eine Handlungseinheit $\mathrm{zu}$ betrachten und diese beiden Delikte in Tateinheit zu stellen.

95 Auch hier ist Tateinheit vertretbar, sofern man das Geschehen als Handlungseinheit betrachtet. 